\title{
An accurate quantification of the flow structure along the acoustic signal cycle in a forced two-phase jet
}

\author{
Esteban Calvo Bernad ${ }^{1, a}$, Juan Antonio García Rodríguez ${ }^{1}$, José Luis Santolaya Sáenz ${ }^{1}$, Luis Adrián Aísa Miguel ${ }^{1}$, J. Ignacio \\ García Palacín ${ }^{1}$
}

${ }^{1}$ Laboratorio de Investigación en Fluidodinámica y Tecnologías de la Combustión-LIFTEC (CSIC-Universidad de Zaragoza), María de Luna, 10, 50018, Zaragoza, Spain.

\begin{abstract}
This paper provides an experimental study of an acoustically forced two-phase air jet generated by a convergent nozzle. The used particles are transparent glass spheres with diameters between 2 and $50 \mu \mathrm{m}$ (which gives Stokes number of order 1) and the selected forcing frequency ( $f=400 \mathrm{~Hz}$ ) induces a powerful nearly periodic flow pattern. Measurements were done by a two-colour Phase-Doppler Anemometer. The experimental setup is computer-controlled to provide an accurate control with a high long-term stability. Measurements cover the whole forcing signal cycle. Raw measurements were carefully post-processed to avoid bias induced by the forcing and the instrument setup, as well as obtain right mean values of the dispersed flow. The effect of the forcing and the particle load allows authors to establish the effect of the acoustic forcing and the particle load on the jet.
\end{abstract}

\section{Introduction}

Two-phase flows have a great interest due to its applications (pharmaceutical and medical uses, industrial and agricultural sprays, combustion, etc.). The dynamics of both phases (dispersed and continuous) are coupled by mass, energy and momentum exchanges. So, the gas (or liquid) drives the particulate phase and particles alter the fluid dynamics. In particular, they modulate the continuous phase turbulence $[1,2]$. In this context, many studies on acoustically forced flows have been done [36]. The acoustic forcing gives a moderate control of the turbulence and the vortex shedding history. In addition, it enhances the ingestion of the surrounding fluid and also roughly controls the particle dispersion. Finally, forcing provides a simplified flow with controlled vortical structures to study the fluid-particle interactions.

Here, an experimental study of a forced two-phase air jet is discussed. Three cases are studied: the unforced jet, the single-phase forced jet and the two-phase forced case. The comparison of these cases provides the effects of forcing and the particle load on the flow.

\section{Experimental methodology}

\subsection{Flow generation and control}

The particle laden forced jet is injected downwards by a pipe of $1 \mathrm{~m}$ in length (fig. 1). The pipe exit has a convergent nozzle with an exit diameter $D_{0}=12 \mathrm{~mm}$ and a contraction ratio of 5.14 to give a top-hat exit velocity profile.

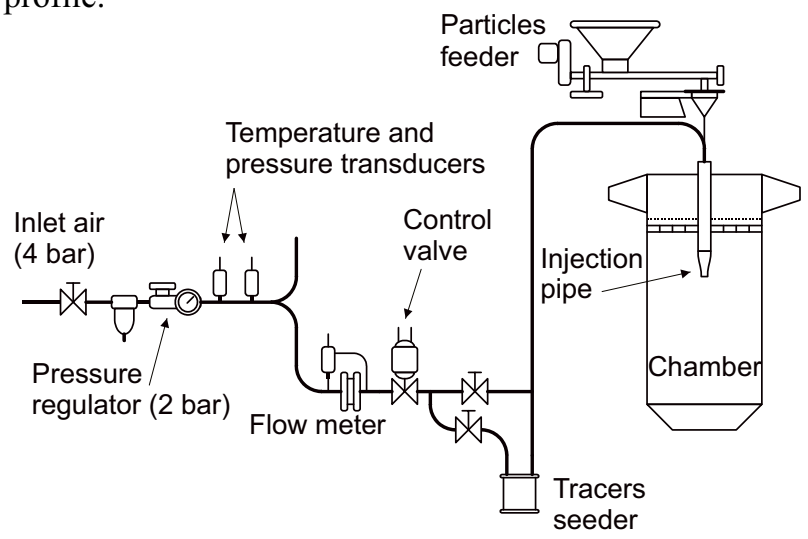

Figure 1. Jet generation setup.

The jet develops at ambient temperature and pressure inside a transparent chamber $\left(480 \times 480 \times 1200 \mathrm{~mm}^{3}\right)$ and it is surrounded by a low velocity co-flow $(0.2 \mathrm{~m} / \mathrm{s})$. The transparent chamber allows the use of optical techniques, as a Phase Doppler Particle Analyzer (PDPA).

An orifice plate and a differential pressure transducer measure the jet flow and a computer controls the air flow by means of an electro-pneumatic valve. The control accuracy is better than $2 \%$.

An $8 \Omega$ speaker forces the jet. It is placed at the upper end of the jet injection pipe. An amplified sinusoidal

\footnotetext{
${ }^{\mathrm{a}}$ Corresponding author: calvober@ unizar.es
} 
signal generator drives the speaker and it also supplies a periodical trigger signal synchronized with the forcing sinusoidal signal. This trigger is useful to measure of the arrival phase of each particle detected by the PDA.

The particles are transparent glass spheres with a mean diameter $D_{10}=25.6 \mu \mathrm{m}$, a rms diameter $\sigma_{D}=11.2$ $\mu \mathrm{m}$ and a density $\rho_{P}=2458 \mathrm{~kg} / \mathrm{m}^{3}$. A rotating screw controls the injected mass rate of particles. A cyclonic device seed the jet with tracers of $\mathrm{Al}_{2} \mathrm{O}_{3}(\sim 0.5 \mu \mathrm{m})$ to measure the gas velocity.

The jet exit velocity is adjusted to $U_{0}=15 \mathrm{~m} / \mathrm{s}$. and the forcing frequency is $f=400 \mathrm{~Hz}$. So, this jet has a Reynolds number $\mathrm{Re}=11900$ and an exit diameter based Strouhal number $\mathrm{St}=f D_{0} / U_{0}=0.32$. This frequency was selected because they cause the powerful perturbation of the jet (Crow \& Champagne. The glass spheres are selected to obtain medium Stokes number (Stk $\sim 1)$. The particle-air mass ratio is $\eta_{M}=0.0704$.

\subsection{Measurement and post-processing}

A two-colour 3100 PDPA of Aerometrics measures the diameter, the radial and the axial velocity of particles crossing the probe volume. Besides, the trigger supplied by the signal generator resets a clock of the PDPA at the beginning of each forcing signal cycle. This clock measures the arrival phase of each detected particle. With this, measurements along the entire forcing signal cycle are done.

Tracers and particles are discriminated using the Doppler-signal intensity and measured diameter. The small $\mathrm{Al}_{2} \mathrm{O}_{3}$ tracers give a low intensity signal and, usually, small diameters.

The flow forcing causes an inhomogeneous tracer seeding till the section $z=10 D_{0}$ and the calculated means of the gas phase could be biased. To avoid this, moments of the overall temporal statistics are obtained from conditional velocity moments for given signal phases $\phi$ :

$$
\begin{gathered}
U=\left\langle U^{\phi}\right\rangle ; \sigma_{u}{ }^{2}=\left\langle(u-U)^{2}\right\rangle=\left\langle\left(U^{\phi}-U\right)^{2}\right\rangle+\left\langle\sigma_{u}{ }^{{ }^{\phi}}\right\rangle \\
\text { with } U^{\phi}=\langle U\rangle^{\phi} ; \sigma_{u}{ }^{{ }^{\phi}}=\left\langle(u-U)^{2}\right\rangle^{\phi}
\end{gathered}
$$

In eq. $1,<>^{\phi}$ is a phase-average and $<>$ is an overall average. Conditional means are not biased since the forced jet is nearly periodic and tracer concentration does not change a lot for a fixed signal-phase.

The shown moments of the particle velocity are massaveraged. Used post-processing removes the bias caused by the particle velocity and the probe volume size. Details can be found in [7]. Velocity means are shown for several particle size classes. Each class has a width of 10 $\mu \mathrm{m}$ and central values $5,15,25 \ldots \mu \mathrm{m}$.

\section{Unforced two-phase jet}

In this section, the behaviour of the unforced jet is discussed. Measurements of the single-phase gas jet (SP jet) and of the dispersed phase are provided. Since the particle-gas mass ratio is small, great changes of the gas dynamics are not expected.
Fig. 2 and 3 show the jet exit conditions. The gas has a top-hat mean velocity profile (fig. 2) with low turbulence intensity (about $3 \%$ and $2 \%$ for the axial and the radial component, in that order). Particles have a smaller axial velocity because the residence time of the glass beads inside the convergent nozzle is smaller than its response time and they do not accelerate as much as the gas.

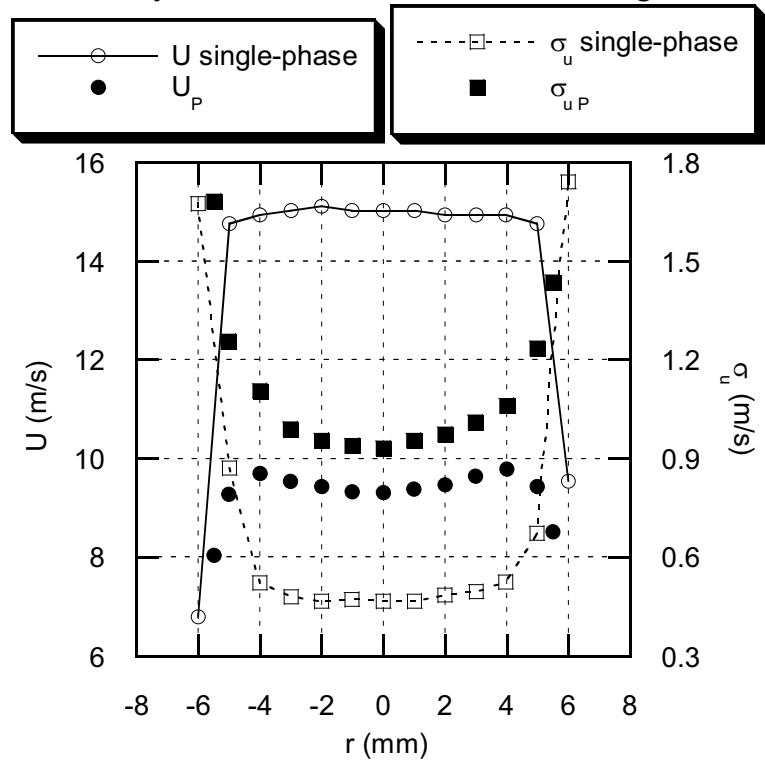

Figure 2. Exit conditions of the gas and particles for the unforced case. $z=0.5 D_{0}$.

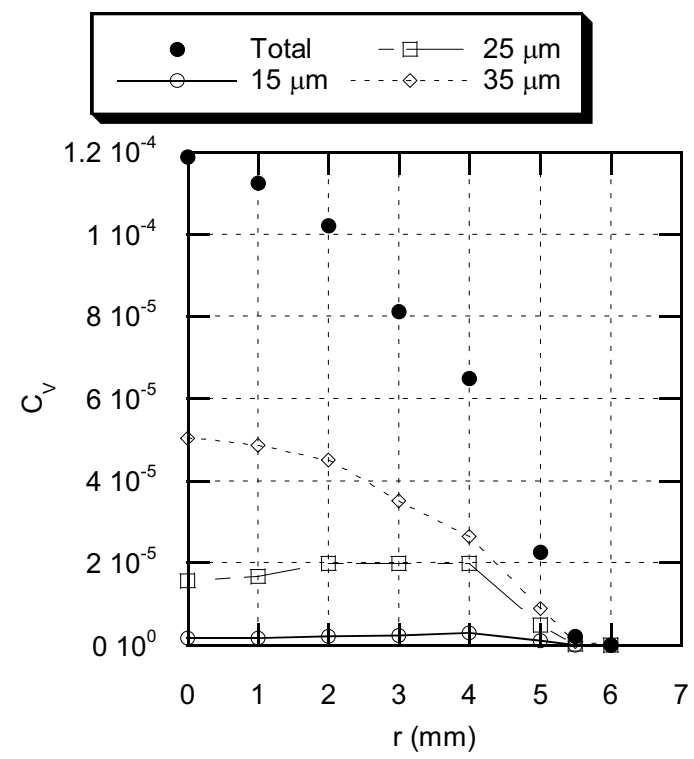

Figure 3. Particle volume concentration. Unforced jet, $z=$ $0.5 D_{0}$.

The dispersed phase focuses around the jet axis (fig. 3) because particles bounce on the nozzle wall. The smaller particles do not focus because they are dragged by the air well after the rebound.

The single-phase gas jet has the usual potential cone and the hyperbolic velocity decay at the jet axis (fig. 4). The linear adjust of the inverse gas velocity in the region $z>25 D_{0}$ gives:

$$
U_{0} / U=0.141\left(z / D_{0}+0.668\right)
$$


The dispersed phase first accelerates because its velocity is smaller than the air velocity and gas drag them. After reach its maximum value $\left(z \sim 6 D_{0}\right)$, the momentum exchange reverse and particles suffer deceleration. In the last measured sections, the particle velocity is close to the gas velocity. The maximum velocity is present at earlier locations for the smallest particles due to its higher exit velocity and its smaller response time. This lets classify the flow in two regions: the zone near the exit (near field, $z<10 D_{0}$ ), where both the gas flow and dispersed flied are evolving, and the far field $\left(z>10 D_{0}\right)$, where the gas almost reach a self-similar flow and the dispersed phase velocity fits to the evolution of the gas velocity.

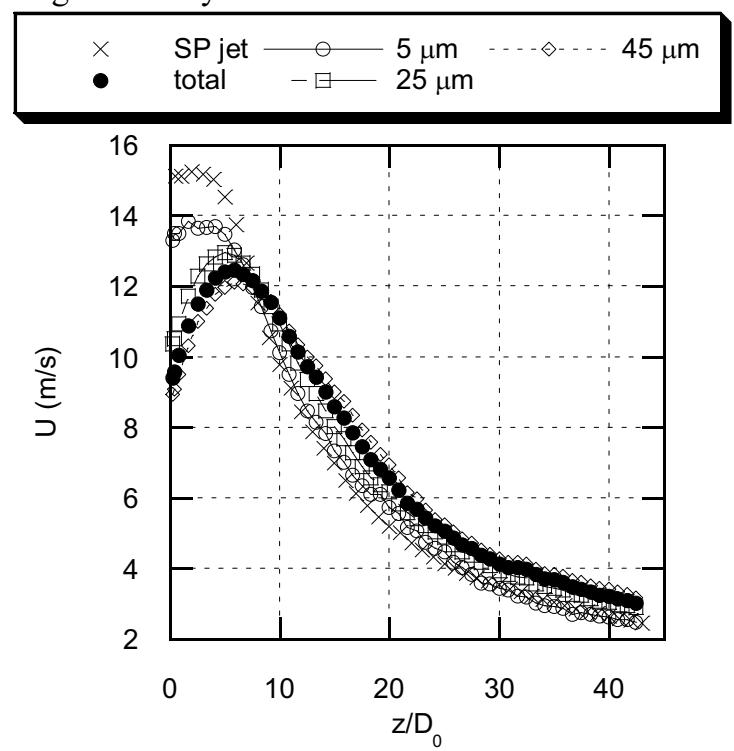

Figure 4. Mean axial velocity of the gas and the particles along the axis. Unforced case.

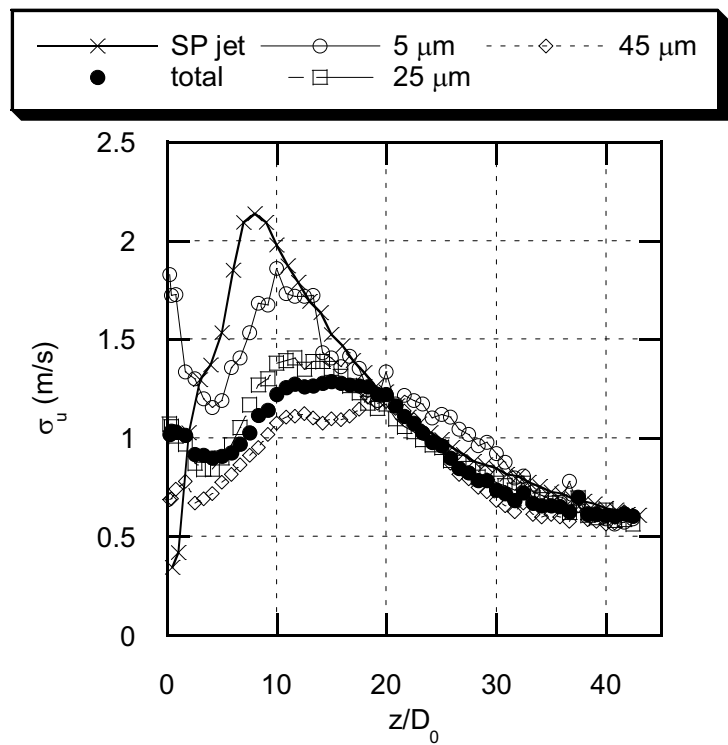

Figure 5. Rms axial velocity of the gas jet and the particles along the axis. Unforced case.

The rms velocity of the particles (fig. 5) hardly reacts to the velocity fluctuations of the gas in the near field and they are controlled mainly by the initial conditions and the inertia. But, downstream, particles respond to the gas turbulence progressively. Again, small sizes adapt before to the gas fluctuations due to its smaller inertia.

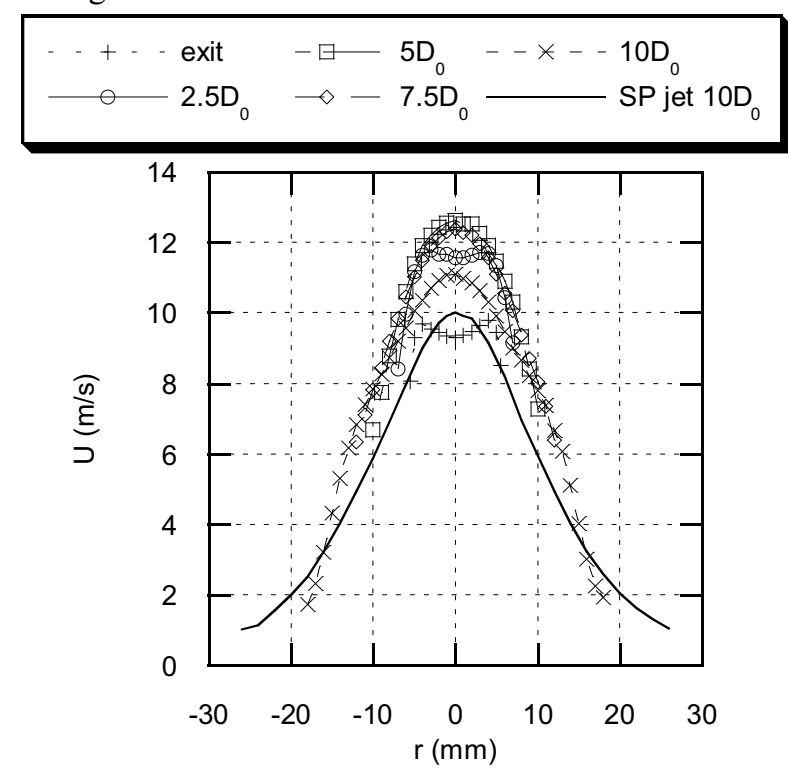

Figure 6. Mean axial velocity profiles of the dispersed phase for several sections of the near field. Unforced case.

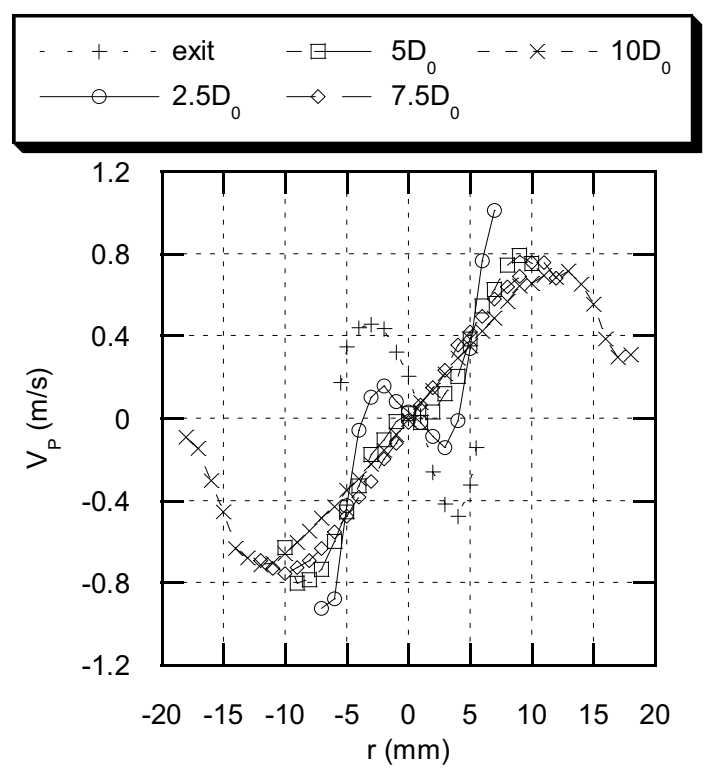

Figure 7. Mean radial velocity profiles of the dispersed phase for several sections of the near field. Unforced case.

The profiles of mean axial velocity (fig. 6) evolves from a nearly flat profile at the nozzle exit (there is a weak minimum at the axis) to a bell-shaped profile at the end of the near field $\left(z=10 D_{0}\right)$. In this section, the particles located in the outer layers are slower in mean that the air. The profiles of mean radial velocity $V$ (fig. 7) shows the particle focusing continues downstream form the nozzle exit. The radial velocity profiles show negative values until $z=5 D_{0}$, so the total mass flux point inwards. The radial zone with $V<0$ becomes narrower when the axial location increases. Downstream section $z=5 D_{0}$, radial velocities are positive and no focusing happens. 

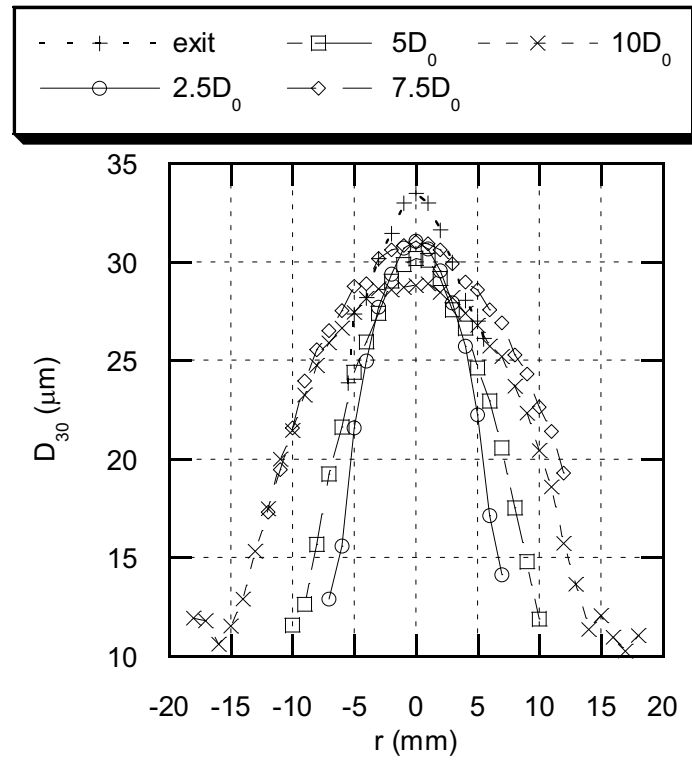

Figure 8. $D_{30}$ profiles for several sections based on number distribution. Unforced case.

The mean diameter $D_{30}$ decreases with the radius (fig. 8). So, the smallest sizes experience an enhanced dispersion since they respond better to the gas flow.

\section{Forced jet}

Now, the main features of the forced jet are discussed. First, results about the single-phase forced jet are shown. After that, the two-phase jet is explored.

\subsection{Single-phase forced flow}

Visualizations of the unforced and forced single-phase flow (fig. 9) show the noticeable changes of the flow field in the near field.
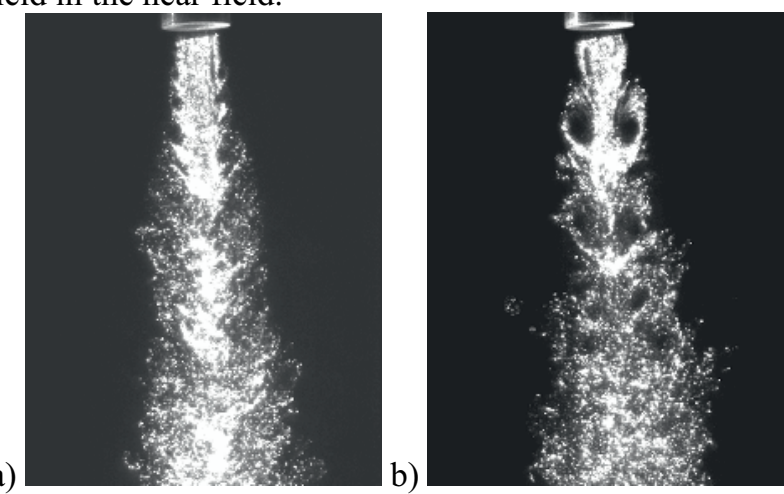

Figure 9. Visualizations of the unforced (a) and forced (b) jet.

The unforced jet develops an axisymmetric mixing layer at the nozzle exit surrounding the potential core of constant velocity. Vortices can be seen inside this mixing layer. The distance between two consecutive vortices roughly scales with the exit diameter but they change over time and, in general, it increases with the axial coordinate.

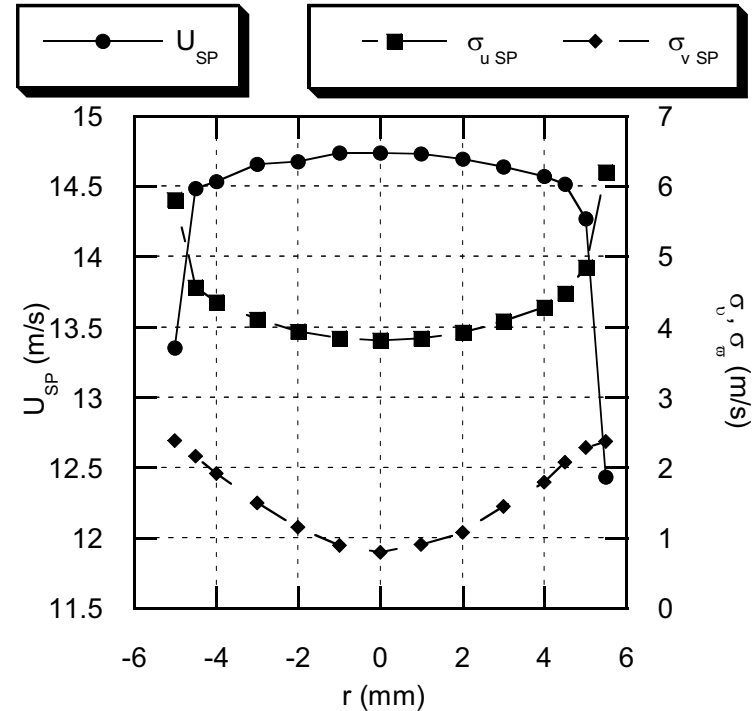

Figure 10. Mean axial velocity and rms of axial and radial velocity for the forced single-phase flow. $z=0.25 D_{0}$.

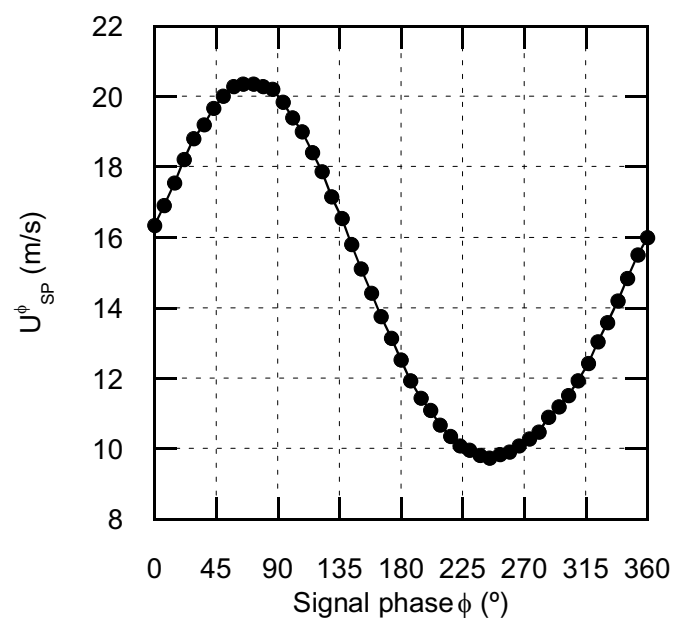

Figure 11. Phase-averaged axial velocity. Forced single-phase case. $z=0.25 D_{0}, r=0$.

The acoustic forcing arranges the near field and flow becomes nearly periodic and coherent with the forcing signal. The vortices inside the mixing layer increase its intensity, emerge periodically from the exit and the distance between them seems to be nearly constant (about $2.2 D_{0}$ ) while they moves downstream. Beside this, other minor secondary vortices are present and some times, this weak vortices pair with the main structures. The coherent flow structure survives until $z=9-10 D_{0}$.

The exit conditions changes too. Mean velocity has too a top hat profile at the jet exit (fig. 10). Forcing induces a powerful sinusoidal velocity wave at the jet exit (fig. 11) along the forcing cycle. This coherent fluctuation is recovered when the total rms velocity is calculated and the rms velocity increase strongly (see fig. 2,10 and 11). 


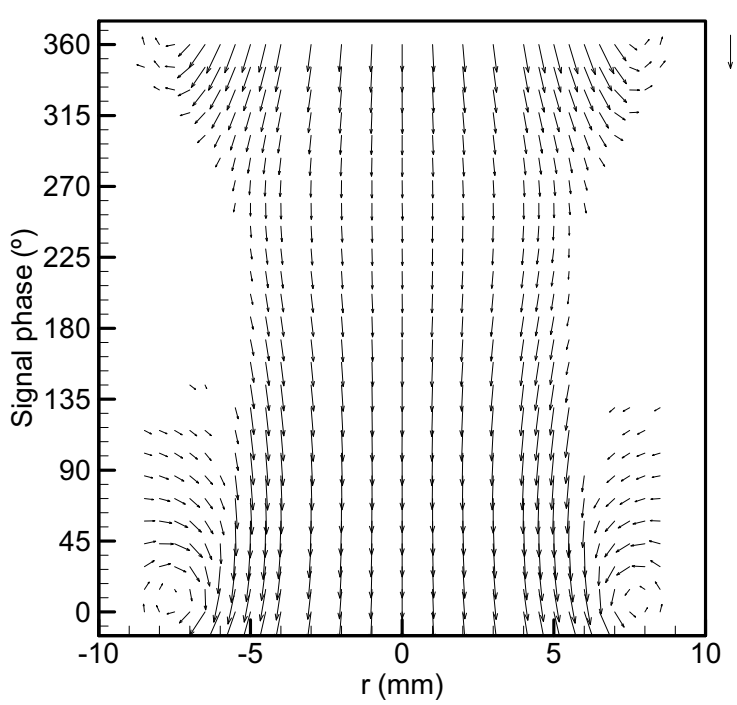

Figure 12. Phase-averaged velocity vs. phase and radius. $z=$ $0.25 D_{0}$. Reference vector $=20 \mathrm{~m} / \mathrm{s}$.

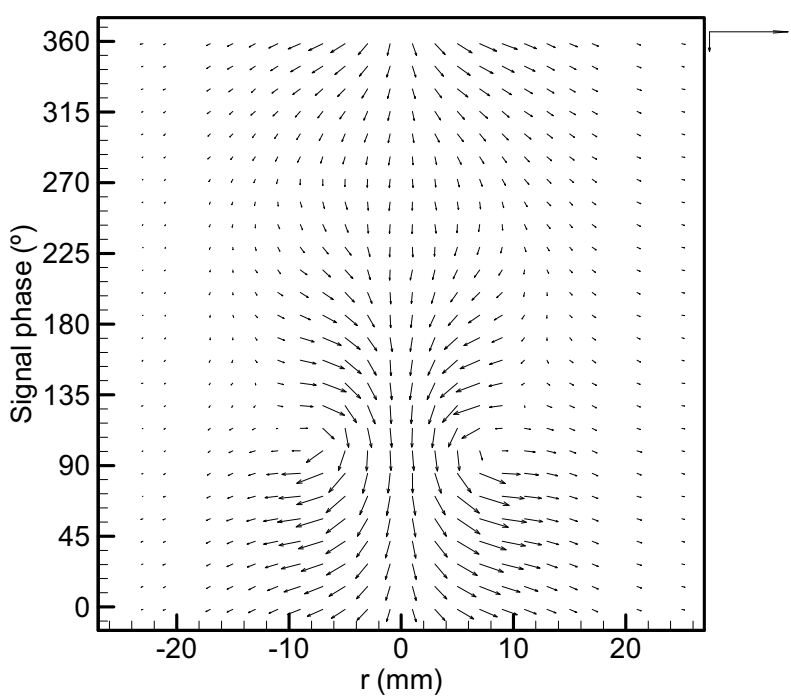

Figure 13. Phase-averaged velocity vs. phase and radius. $z$ $=5 D_{0}$. Reference vectors $=20 \mathrm{~m} / \mathrm{s}$.

Fig. 12 and 13 plot the phase-averaged air velocity for $z / D_{0}=0.25$ and 5 , in that order. In these plots, signal phase can be thought as a pseudo-spatial axial coordinate and the vortex emerges clearly. The vortex core is located about $r \sim 7 \mathrm{~mm}$, outside of the exit nozzle edge.

This periodic flow structure changes the jet development along its axis. The potential core of the unforced jet ( $z<5 D_{0}$ with constant velocity at the axis) is replaced by a zone with decreasing axial velocity (fig. 14). At the end of the near field, the forced case has a smaller velocity than the unforced jet. For the forced jet, the linear adjust of the inverse gas velocity along the axis for $z>25 D_{0}$ gives:

$$
U_{0} / U=0.149\left(z / D_{0}+4.16\right)
$$

Comparing with eq. (2), the virtual origin of the forced jet moves upstream and has a slightly decaying slope.

Rms velocity of the forced case is higher in the near field $\left(z<10 D_{0}\right)$ because here the flow has powerful coherent velocity fluctuations (fig. 14 and 9). As visualizations suggest, coherent structures weaken downstream and the forced flow recover the rms velocity level of the unforced case. Comparing the nondimensional rms velocity (fig. 15), the forced case reach the self-similarity for the second velocity moments at early sections $\left(z \sim 20 D_{0}\right)$ while the non-dimensional rms velocity of the unforced jet is still growing.

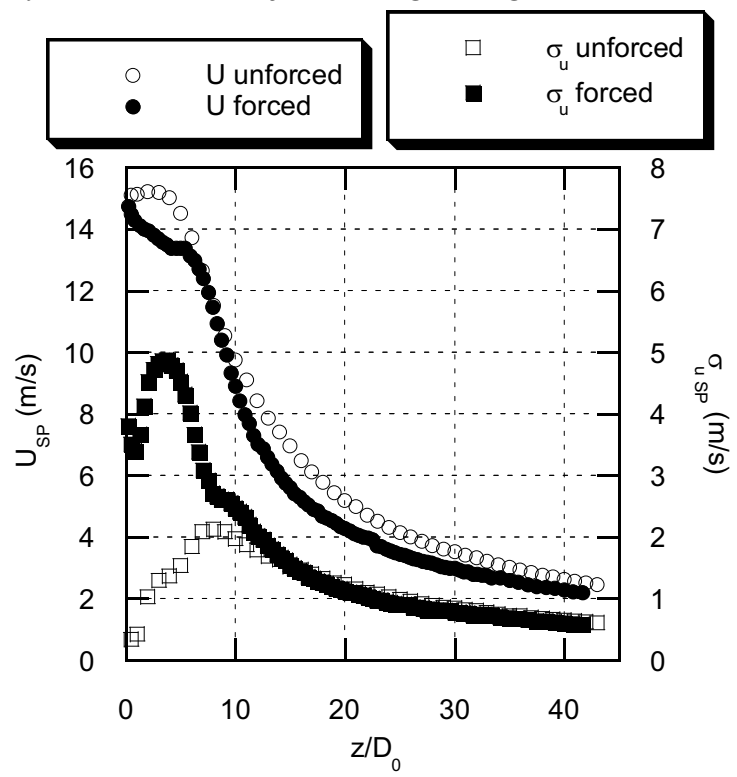

Figure 14. Mean and rms of axial velocity along the jet axis. Forced and unforced single-phase flow.

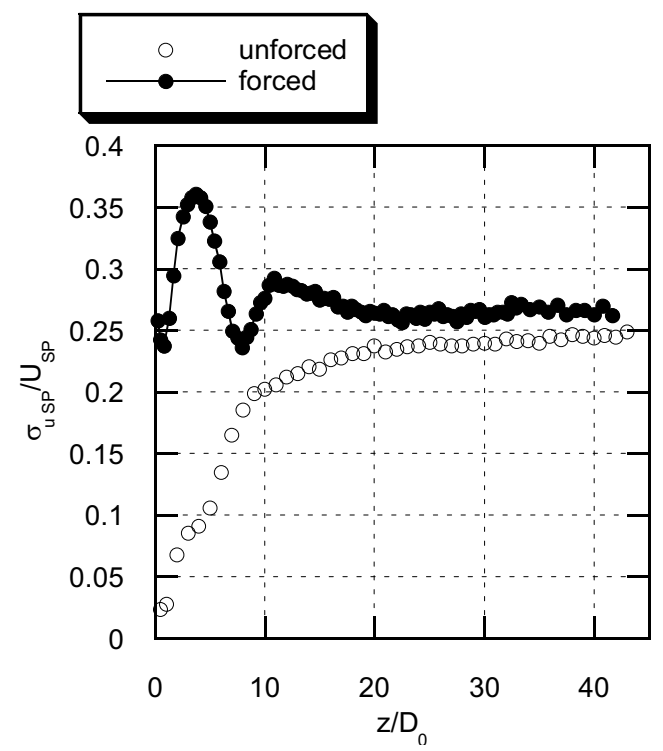

Figure 15. Non-dimensional rms axial velocity at the jet axis. Forced and unforced air jet.

\subsection{Two-phase forced flow}

Fig. 16 shows the exit conditions of the forced two-phase jet for the velocity of both phases. The mean velocity profiles are similar to the ones of the unforced jet. However, the rms velocity markedly increases due to the forcing.

Concentration of small particles at the exit (fig. 17) has a maximum in the outer radii and higher sizes, as in the unforced jet, but the highest sizes shown a flatter concentration profile (see fig. 3 ). 


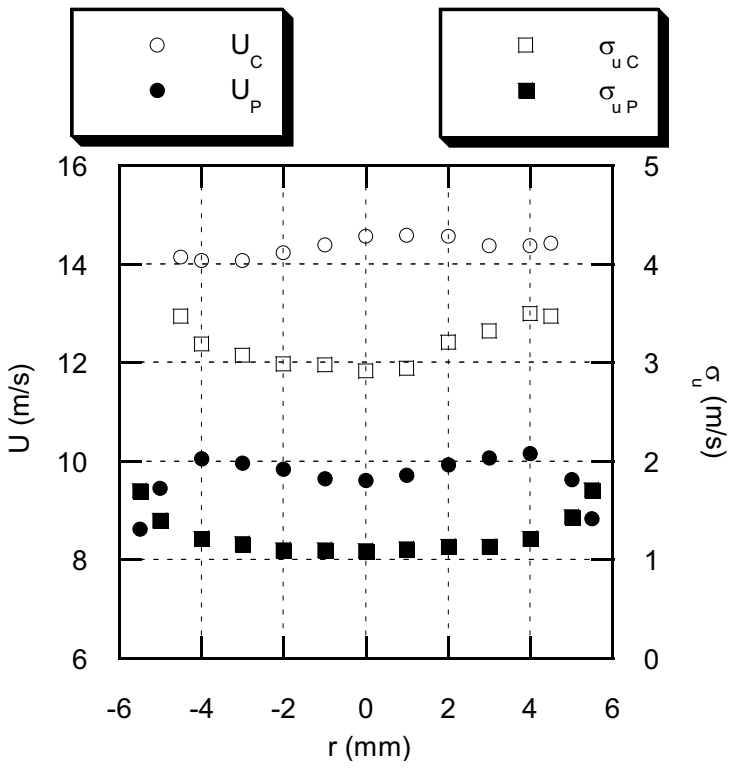

Figure 16. Exit conditions of the continuous $(C)$ and the dispersed $(P)$ phase. Forced case, $z=0.25 D_{0}$.

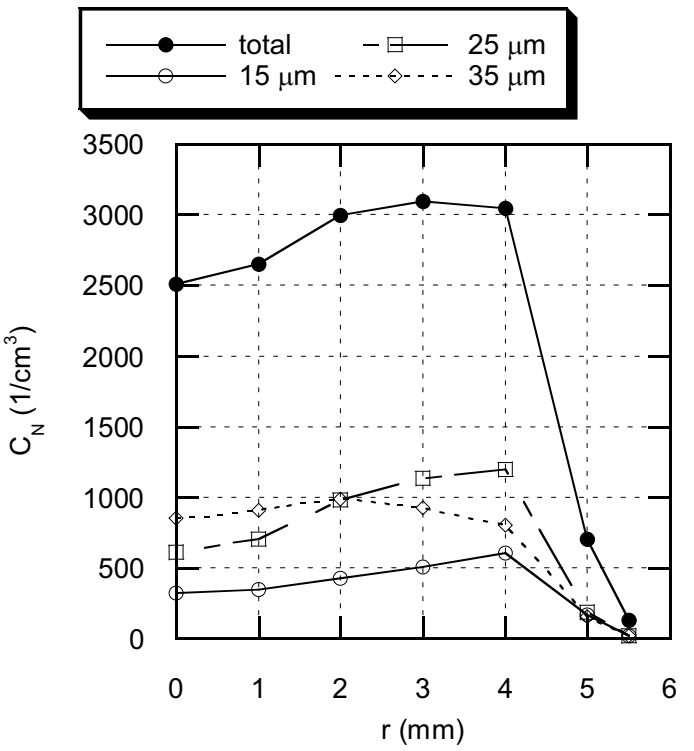

Figure 17. Particle concentration profiles. Forced case, $z=$ $0.25 D_{0}$.

When glass beads are added to the flow, changes are observed. While the change of the mean velocity is not relevant, the rms values are smaller at the near field (see the development of the gas movement along the axis, fig. 18). Downstream, both the single-phase and the continuous phase have similar turbulence levels.

In fact, particles weaken the coherent structure of the gas flow. It can be measured by two ad-hoc defined variables: the Modulation $(M)$ and the Coherence $(C)$ of the axial velocity. They are defined as:

$$
M=\left\langle\left(U^{\phi}-U\right)^{2}\right\rangle^{1 / 2} ; C=M / \sigma_{u}
$$

Modulation is the rms value of the phase-averaged mean velocity and it measures the power of the velocity wave coherent with the forcing signal. Eq. 1 gives sense to the coherence. The total velocity variance $\sigma_{u}{ }^{2}$ is the sum of the square of modulation $\left(M^{2}\right)$ and the mean value of the phase-averaged variance $\left(\sigma_{u}{ }^{2}\right)$. Phase-averaged variance is caused by the turbulence (incoherent with the forcing). So, coherence is 1 when there is no turbulence and it is 0 when there is no coherent flow structure.

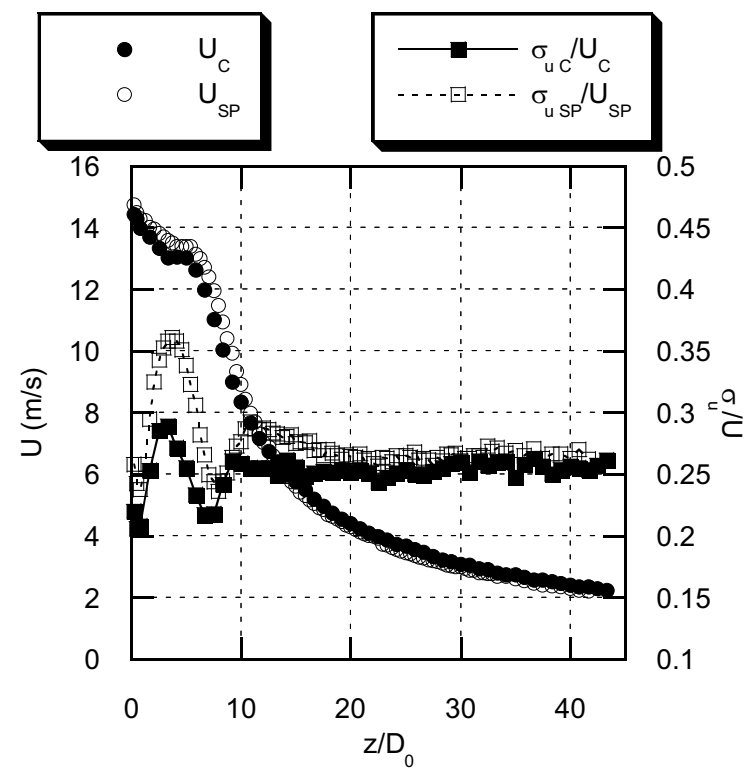

Figure 18. Mean and non-dimensional rms axial velocity at the jet axis. Forced case, single-phase jet (SP) and continuous phase (C) of the two-phase flow.

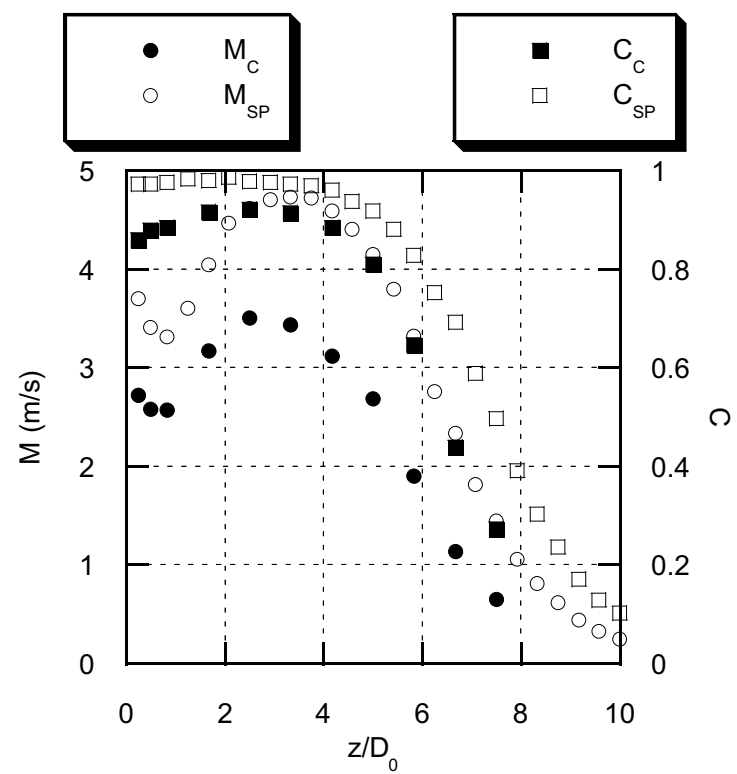

Figure 19. Modulation and Coherence along the axis. Singlephase jet (SP) and continuous phase (C)-

The coherent structures of both flows have similar evolutions (fig. 19). Modulation saturates at $z \sim 3 D_{0}$ and the potential core region of the unforced jet $\left(z<5 D_{0}\right)$ retain its main feature: it is a region with low turbulence intensity. But the continuous phase has weaker coherent vortices and they dissolve earlier since the coherence of the two-phase case goes to 0 faster. So, the dispersed phase inhibits the presence and growth of the coherent vortices.

The evolution of the mean axial velocity of the particles (fig. 20) shows trends similar to the unforced case: particles accelerate in the near field due to the air drag and downstream, they decelerate since they transfer 
momentum to the gas until both phases have similar velocities. The smallest sizes react quicker to the gas flow due to its smaller response time.

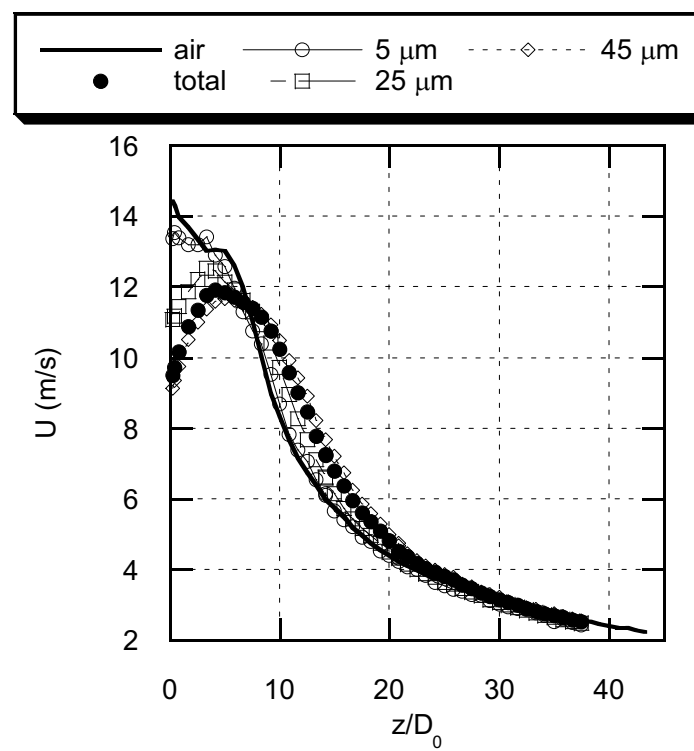

Figure 20. Mean axial velocity of both phases along the axis. Forced jet.

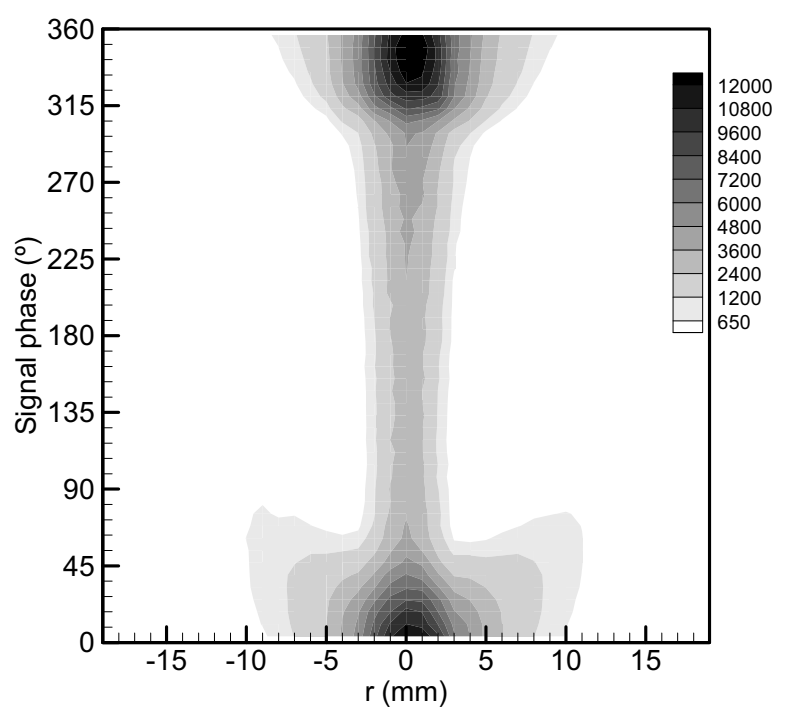

Figure 21. Total particle number concentration vs. radius and phase. $z=5 D_{0}$.

The dispersed phase also has a coherent structure along the forcing signal cycle since particles are driven by the structured gas flow. The first consequence is the formation of high-concentration particle clusters in the near field. These clusters are coherent with the forcing signal and the coherent air vortices.

Measured particle concentration vs, radius and phase in $z=5 D_{0}$ (the end of the high coherence zone, fig. 21) shows how vortices concentrate effectively particles in some phases of the forcing signal. Phases with maximum concentration match with the phases of smallest axial velocity. An axi-symmetric streak or "tongue" of particles dragged by the air emerges from this maximum. Two successive clusters are connected by a stretched column of particles. Inside this column, air movement concentrates the particles.

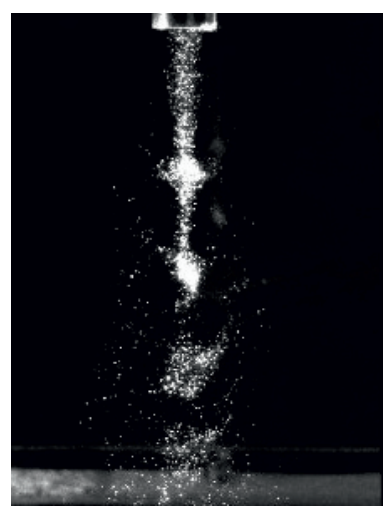

Figure 22. Visualization of the dispersed phase. Forced case.

Downstream $z=5 D_{0}$, the continuous phase loss its coherent periodic movement, but particles retain coherency with the forcing signal due to its inertia. In particular, clusters survive until advanced sections (see the visualization in fig. 22). At about $z \sim 10 D_{0}$, clusters begin to reconnect to finally dissolve.

\section{Effect of forcing on particle dispersion}

Acoustic forcing greatly enhances the particle dispersion at the end of the near flow field. Fig. 24 plots the percentage of the particle mass rate which passes inside the radius $r$ in the section $z=10 D_{0}$. While most of the particle mass pass inside $r=10 \mathrm{~mm}$ for the unforced case, it spreads beyond $r=30 \mathrm{~mm}$ when forcing is enabled. So dispersion is enhanced by a factor three.

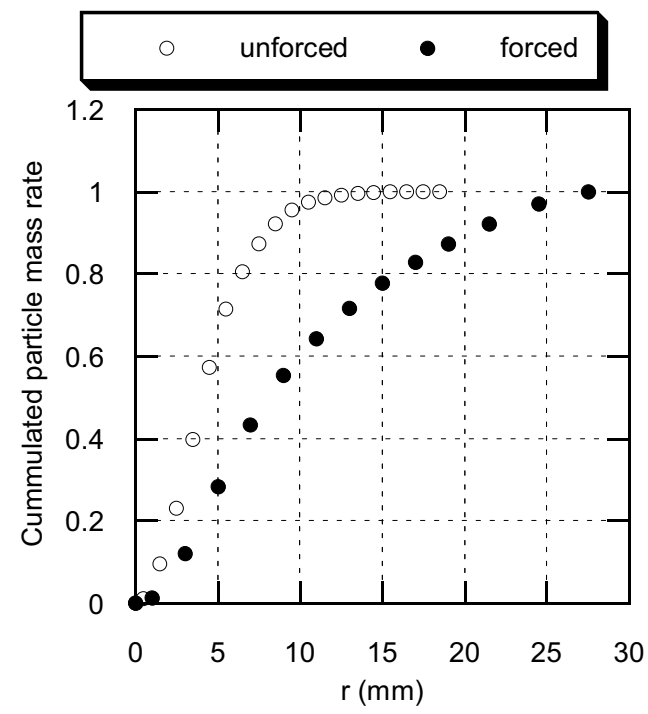

Figure 23. Particle mass flow inside a circle of radius $r . z=$ $10 D_{0}$.

\section{Conclusions}

A forced turbulent two-phase jet seeded with glass spheres has been tested extensively. Experiments conditions (Reynolds number, forcing frequency and particle sizes) have been selected to obtain a particle Stokes number around 1. Measurements have been made using a Phase Doppler Particle Analyzer (PDPA). A careful post-processing of the raw data supplied $y$ the 
instrument has been done to avoid biases and obtain right statistical moments of the dispersed phase (as concentration, velocity moments, etc.). Measurements cover the entire forcing signal cycle.

Three cases are compared: the unforced two-phase jet, the forced single-phase gas jet and the forced two-phase jet. This lets evaluate the effect of particles and forcing in the flow.

Forcing induces a nearly periodic structure coherent with the excitations signal. Coherent vortices emerge from the nozzle edge. The coherent flow structure is notable inside the older potential core of unforced case and it disappears progressively in $z \sim 10 D_{0}$. After that, a selfsimilar jet develops quickly while the unforced jet is still evolving towards this state.

Particles weaken the periodical flow structures and promote their earlier dissolution. The dispersed flow is also affected by the gas coherent structure. The gas motion and particle inertia promote the particle clustering. The clusters are fully developed just downstream of the fully coherent gas flow and retains some degree of coherent structure till $z \sim 10 D_{0}$ due to its inertia.

Finally, acoustic forcing promotes the enhanced particle dispersion. The outermost radius with presence of particles increases by a factor 3 .

\section{References}

1. R.A. Gore, C.T. Crowe, Int. J. Multiphase Flow 15, 279-285 (1989)

2. P. Gualtieri, F. Picano, G. Sardina, C.M. Casciola, J. Fluid Mech., 715, 134-162 (2013)

3. S:C: Crow, F.H. Champagne, J. Fluid Mech. 48, 547-591 (1971)

4. R.A. Petersen, M.M. Samet, J. Fluid Mech., 194, 153-173, (1988)

5. E.K. Longmire, J.K. Eaton, J. Fluid Mech., 236, 217 257 (1992)

6. B.J Lázaro, J.C. Lasheras, J. Fluid Mech. 235, 179221, (1992)

7. E. Calvo, J.A. García, J.L. Santolaya, I. García, L. Aísa. Meas. Sci. Technol. 23, 055202 (2012) 